\title{
Adjustable sutures in eyelid surgery for ptosis and lid retraction
}

\author{
J R O Collin, B A O’Donnell
}

\begin{abstract}
New techniques are described and illustrated for ptosis and lid retraction surgery in which the sutures holding the upper eyelid position are adjustable postoperatively. In the anterior approach, the sutures pass from the levator muscle through the anterior surface of the tarsal plate at the position of the skin crease and are tied at the skin crease incision. In the posterior approach, the sutures pass from the levator muscle through the cut upper edge of the tarsal plate and are tied at the position of the desired skin crease. The techniques allow the lid height to be adjusted for over and undercorrection medially, centrally, laterally, or overall if required, thereby achieving the optimal surgical result without altering the position of the skin crease. Suture adjustment
\end{abstract}

Retractor recession

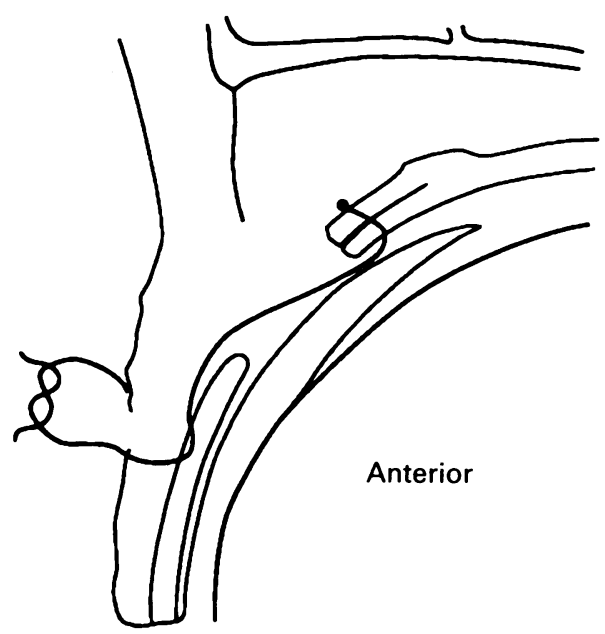

Figure 1A Anterior approach: upper lid lowering/retractor recession. is a simple procedure and is recommended at 24 hours.

(Brf Ophthalmol 1994; 78: 167-174)

The majority of operations to correct upper lid retraction or ptosis are successful. However, the results are not always predictable with part or all of the lid at an unsatisfactory height postoperatively. With ptosis surgery, the lid height may be relatively easily lowered by a minor second procedure and lid traction if posterior approach surgery was used, but the amount of postoperative correction is limited. Surgeons less familiar with the posterior approach technique do not have this advantage. Following the more commonly performed anterior approach surgery, if the postoperative lid height is unsatisfactory, the levator must be reset with new sutures and this revision is more complicated.

With upper lid lowering for lid retraction,

Levator resection

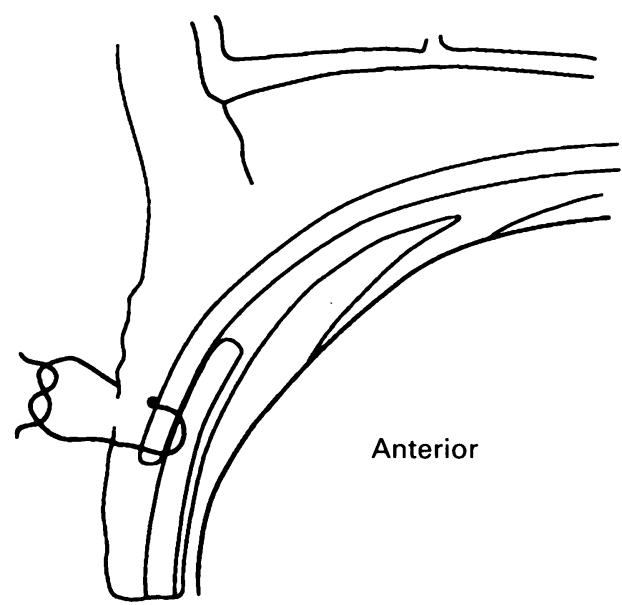

Figure 18 Anterior approach: upper lid ptosis correction/ levator resection.
Oculoplastic Service, Moorfields Eye Hospital J R O Collin

B A O'Donnel

Correspondence to:

Mr Richard Collin, Moorfields

Eye Hospital, City Road,

London ECIV 2PD.

Accepted for publication 28 July 1993

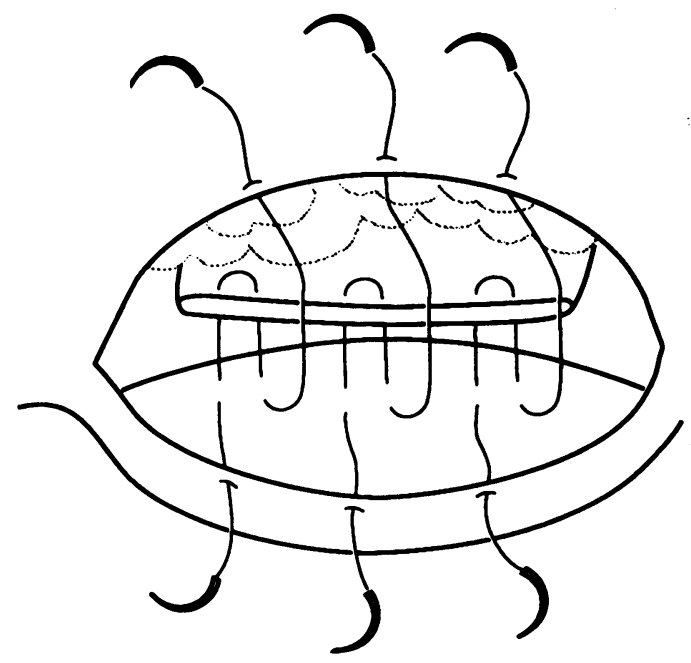

Figure 1C Anterior approach placement of sutures for lid lowering or elevation. 
Retractor recession

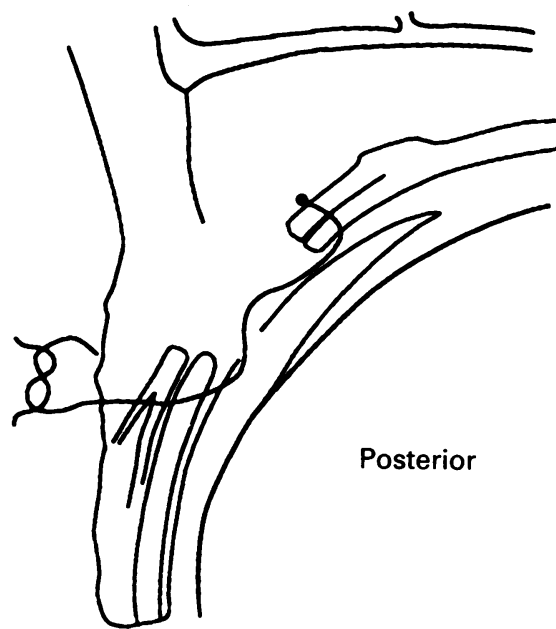

Figure 2A Posterior approach: upper lid retractor recession.

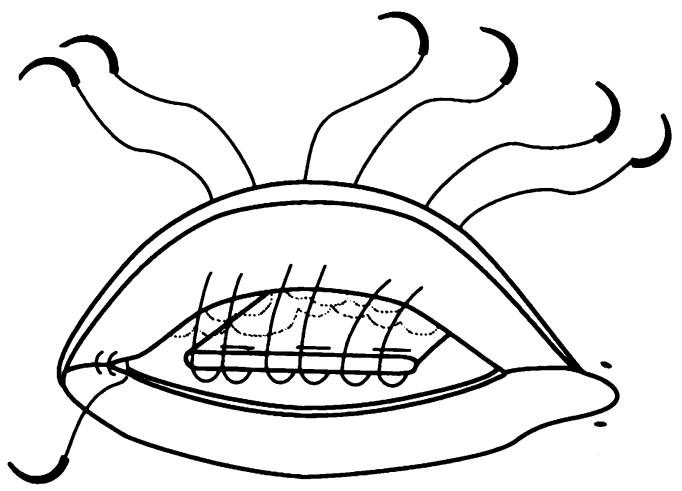

some adjustment in the postoperative lid height is possible with the use of reverse Frost sutures placed in the upper lid margin and taped to the cheek for 24 to 72 hours. The lid height may be inspected at 1, 2, and 3 days, and the reverse Frost suture removed if the lid appears overcorrected. The results of surgery for thyroid lid retraction are frequently unpredictable postoperatively with problems of persisting lateral flare, and over and undercorrection. A means of postoperative lid adjustment would frequently be very desirable.

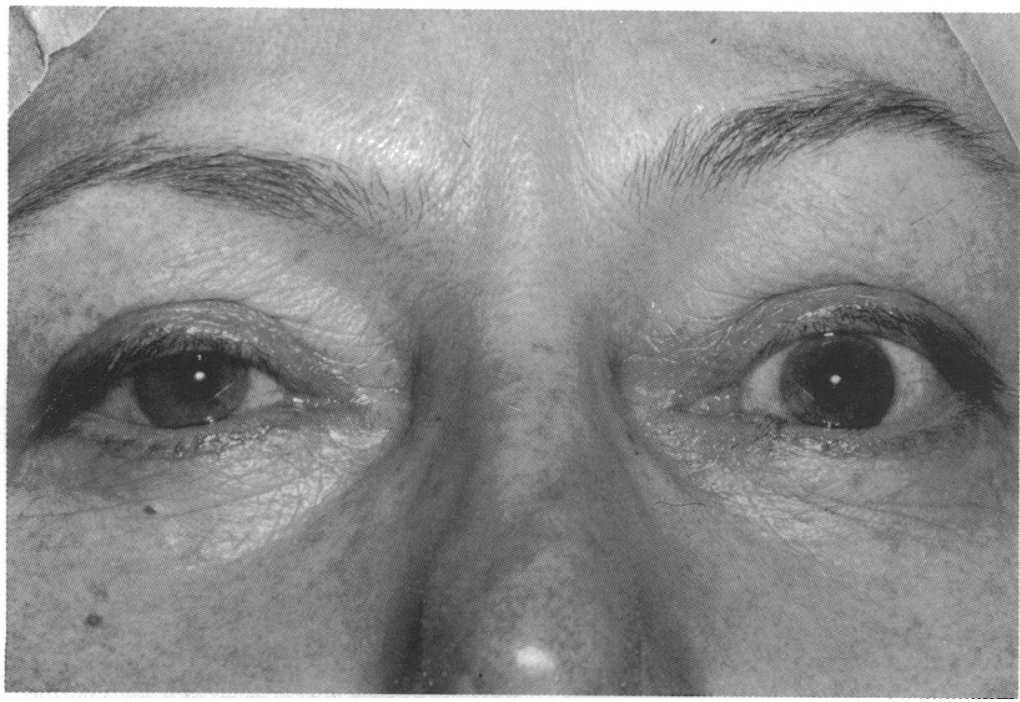

Figure $3 A$ Preoperative photograph of left upper lid thyroid lid retraction.
Levator resection

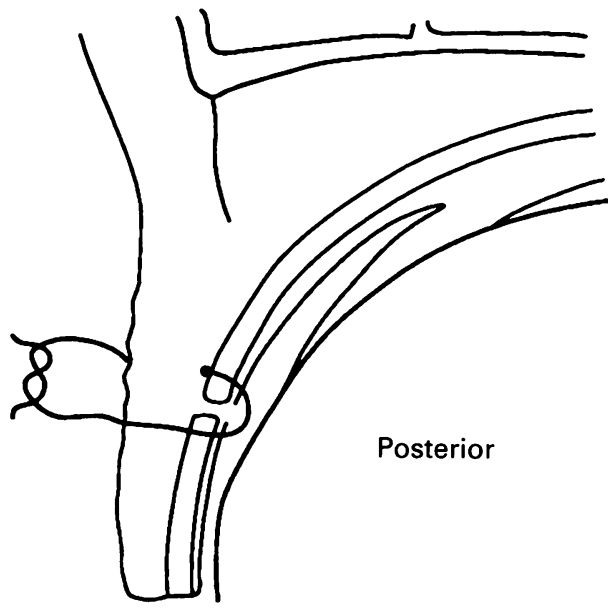

Figure 2B Posterior approach: upper lid ptosis correction.

Figure 2C Posterior approach placement of sutures for lid lowering and elevation showing conjunctival closure with running suture.

The following describes techniques for anterior and posterior surgery for lid retraction and ptosis. The techniques are new with postoperative adjustment of the lid height possible.

\section{Material and methods}

TECHNIQUE FOR ANTERIOR APPROACH ADJUSTABLE EYELID SUTURES FOR CORRECTION OF LID

RETRACTION AND PTOSIS (FIGS 1A, B, C)

Routine anterior approach surgery is performed to expose/mobilise the upper lid retractor muscles. ${ }^{1}$ The skin crease is marked at the desired height with a skin marking pen and an incision is made through skin and orbicularis. The dissection is continued to expose the tarsal plate. The septum is opened, the aponeurosis is exposed, and the preaponeurotic fat pad is released as required. In the correction of lid retraction, the lateral horn is cut and the medial horn weakened as required. The levator muscle may be isolated and a $\mathrm{z}$-myotomy performed for additional recession. Whitnall's ligament may need to be weakened. Dissecting the levator and Muller's muscles off the common sheath will further recess the upper lid retractors. In ptosis correction, the levator aponeurosis, levator muscle, or levator/Muller's complex is mobilised. The horns are not usually cut. Preaponeurotic fat is freed as necessary. If a levator resection is required, the aponeurosis is dissected off Muller's muscle to $10-12 \mathrm{~mm}$ above the tarsal plate, the origin of Muller's muscle. 
Figure 3B Anterior approach showing mobilisation of levator muscle and exposed anterior surface of tarsal plate.

Figure 3C Suture in position passing from levator through tarsus and exiting via superior and inferior skin edges.

Figure 3D Three sutures in position.

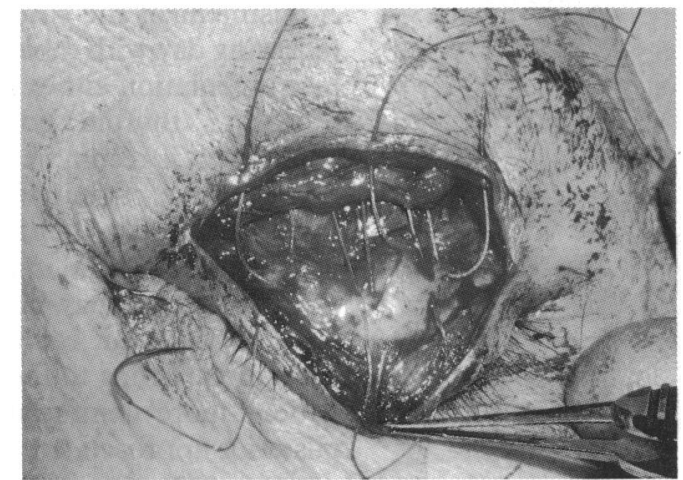

This is then incised to expose common sheath/ upper fornix, and blunt spreading with scissors proximally will mobilise the levator muscle from the underlying common sheath. Alternatively, Muller's muscle and levator aponeurosis may be dissected as a single layer from the conjunctiva. The former method will leave Muller's muscle intact and functional postoperatively. Three or four double armed 5/0 polyglycolic acid sutures are placed through the levator aponeurosis/ upper lid retractor complex. These are then passed in and out through the anterior surface of the tarsal plate at the height of the desired skin crease. This bite of the anterior surface should be deep (but not full thickness) to prevent pull out when adjusting. One end of each suture is then passed through the upper edge of the skin incision and one end through the lower edge. A single throw then bow knot is tied after setting the lid height. This suture also closes the wound. Additional sutures may be needed for skin/ orbicularis closure. Intraoperative haemostasis should be meticulously carried out and firm padding is recommended to minimise postoperative oedema. The lid sutures are only tied when the surgeon is satisfied with the position of the lid. Thus, adjustable sutures are not an alternative to careful surgery, but should be used in conjunction with the surgeon's usual intraoperative efforts to set the lid at the correct height.

\section{TECHNIQUE FOR POSTERIOR APPROACH}

ADJUSTABLE EYELID SUTURES FOR CORRECTION OF LID RETRACTION AND PTOSIS (FIGS 2A, B, C)

Routine posterior approach surgery is performed to expose and/or mobilise the upper lid retractor muscles. ${ }^{1}$ For the correction of ptosis, the position of the skin crease is marked on the skin, then the lid is everted over a Desmarres retraction. An incision within $1 \mathrm{~mm}$ of the skin crease height is made in the tarsal plate. For the correction of lid retraction, the incision is made at the superior edge of the tarsus. In both cases, the proximal cut edge is retracted and dissection is performed posterior to the levator aponeurosis to expose the 'white line', where the levator aponeurosis is reflected back on itself. This is incised transversely to expose the anterior surface of the aponeurosis and levator muscle. The position of the Desmarres retractor is altered to retract the upper lid and preaponeurotic fat pad, which is freed as necessary. In correction of lid retraction, the lateral horn of the levator is cut and the medial horn is weakened as required. The levator muscle may be isolated and a $\mathrm{z}$-myotomy can be performed. Dissection of the levator and Muller's muscles off the common sheath may further recess the upper lid retractor muscles. Whitnall's ligament may need to be divided. In correction of ptosis, the levator aponeurosis, levator muscle, or levator/Muller's complex is mobilised and the levator muscle resected as appropriate and as discussed for anterior approach ptosis surgery.

Three or four 5/0 polyglycolic sutures are placed through the levator aponeurosis. The sutures are then passed through the edge of the tarsal plate and through the skin at the position 
of the previously marked skin crease for ptosis correction. For the correction of lid retraction, the sutures are brought out through the upper border of the tarsal plate and through the skin at the same level. The conjunctival incision is

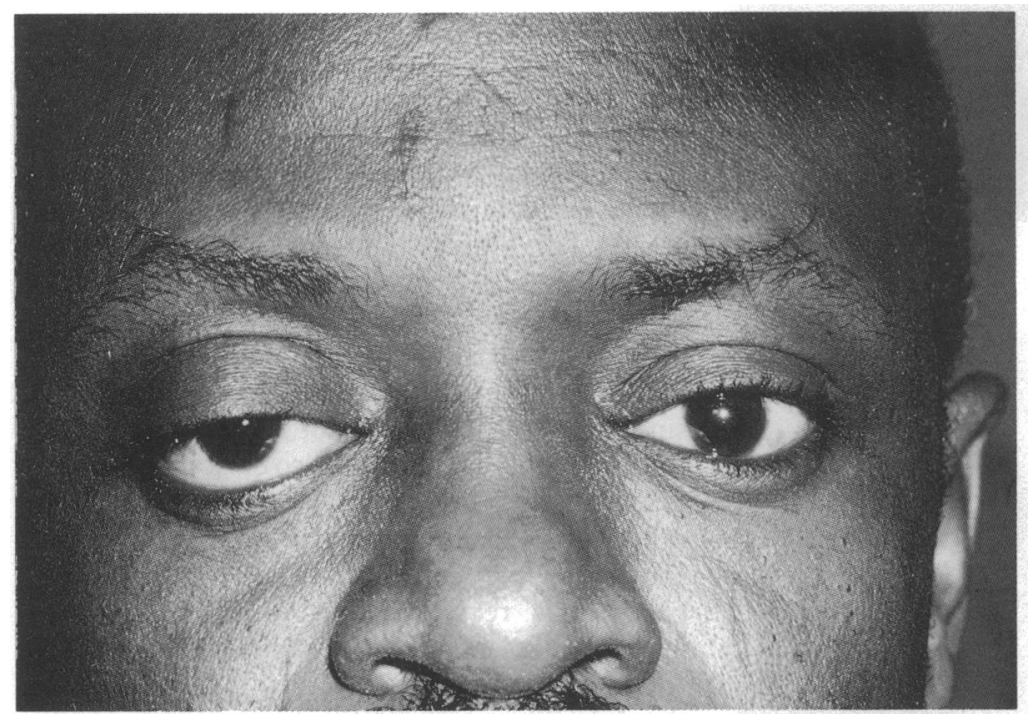

Figure 4A Preoperative photograph of right upper lid ptosis.

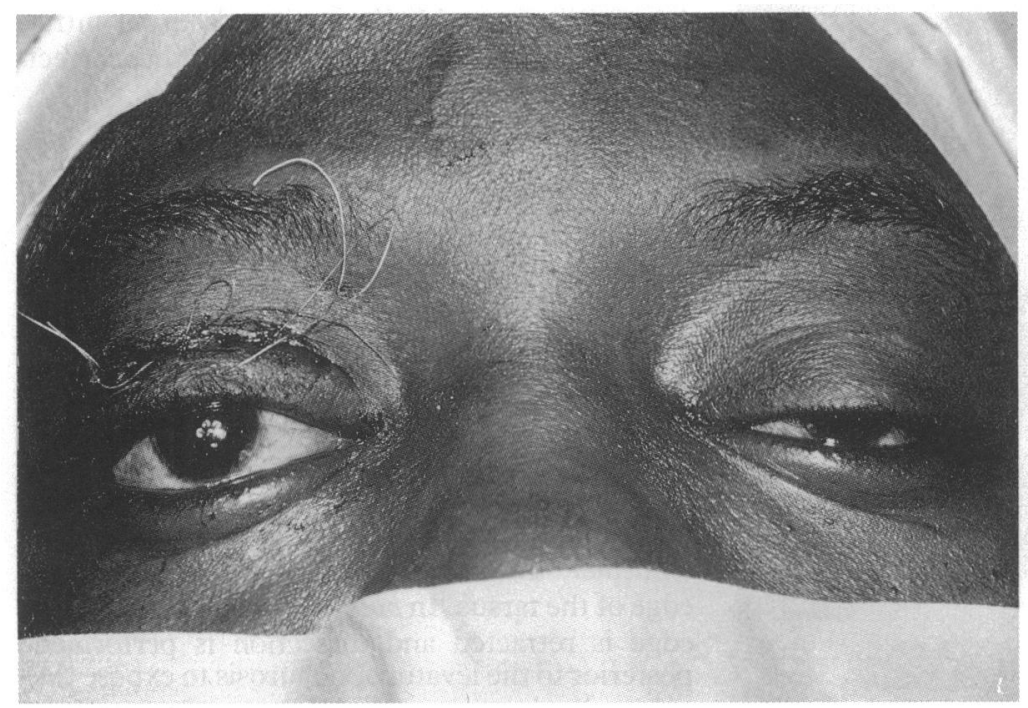

Figure 4C Intraoperative lid height set.

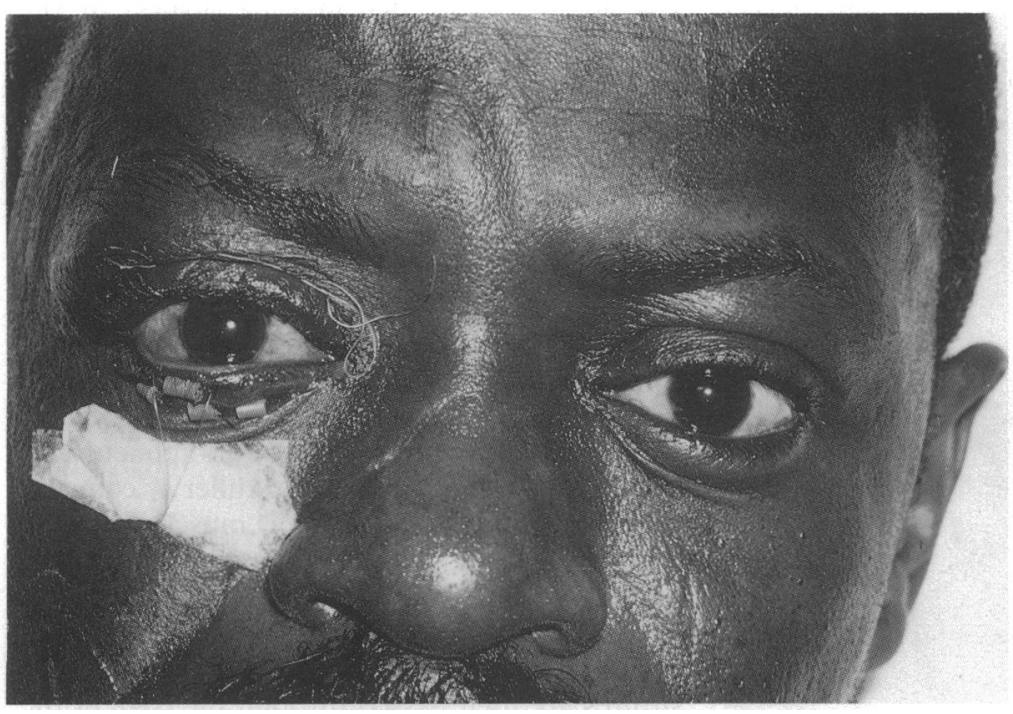

Figure 4D Lid height after small postoperative adjustment.

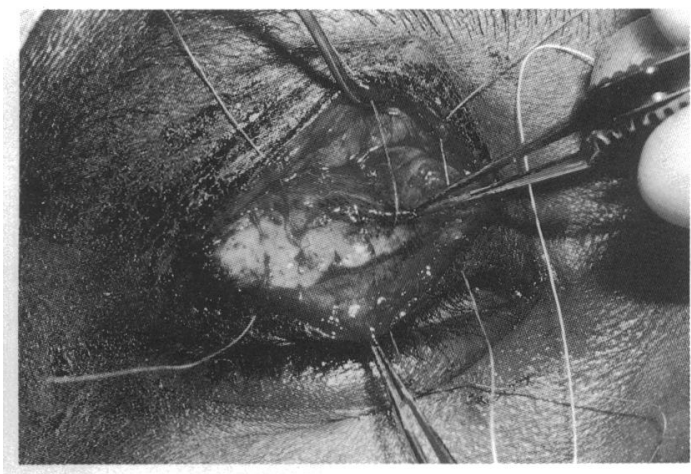

Figure 4B Three sutures in place.

closed with a running, 6/0 plain cat gut suture, taking care to bury the knots.

Alternatively, a running, 6/0 nylon pullout suture can be used and passed through the skin at each end of the incision where it is taped. A single throw then bow knot is tied with the $5 / 0$ suture over a bolster.

Operative photographs illustrate anterior approach correction of lid retraction (Figs $3 \mathrm{~A}-\mathrm{E}$ ) and ptosis (Figs 4A-D); and posterior approach correction of thyroid lid retraction (Figs $5 A-I$ ). Topical chloramphenicol antibiotic drops and ointment are routinely prescribed.

\section{POSTOPERATIVE MANAGEMENT}

Adjustment of the sutures is performed on the following day with topical anaesthetic and sterile instrumentation either in the ward or surgeon's office. In thyroid lid retraction and ptosis, adjustment is recommended within 24 hours. At this time the patient will be alert and cooperative. Adjustment after this would allow more lid oedema to settle and muscle function to recover, but the sutures may be more difficult to adjust since tissue repair is already occurring. Topical anaesthetic drops to the globe usually make the patient more comfortable and are essential if posterior approach surgery was performed. The patient is seated or prone, and the eyelid adjusted as required. The eyelid can be raised by simply tightening the sutures and lowered by loosening the sutures, holding the eyelashes and requesting the patient to look up. After each adjustment, the patient is seated and asked to look up and down so that the lid retractors reposition themselves. When the lid position is satisfactory, the sutures are securely tied. In lid retraction, a reverse Frost suture is recommended to hold the lid closed and is taped to the cheek so that the upper lid retractors are in the recessed position in the early postoperative period. If a running $6 / 0$ nylon suture has been used to close the conjunctiva, it is removed after 5 days.

\section{Results}

Adjustable sutures have been used successfully for aponeurotic repair and levator resection in ptosis by both anterior and posterior approaches and for the correction of lid retraction, also by the anterior and posterior approaches in 15 patients. There were eight patients with ptosis. The causes were post-traumatic (three), post- 
Figure 5A Posterior approach incision is made through conjunctiva and Muller's muscle at the upper border of the tarsus for the correction of lid retraction.

\section{Figure $5 B$ The} preaponeurotic fat pad is exposed and the cut edge of the aponeurosis is visible (arrow).

Figure 5C Three 5/0 sutures are placed through the edge of the aponeurosis/ levator muscle.

Figure 5D A suture is placed through the upper edge of the tarsal plate, then through the skin at the position of the skin crease.

Figure 5E Lid still everted over Desmarres retractor showing posterior surface of the lid with all three sutures in place but not yet tied.

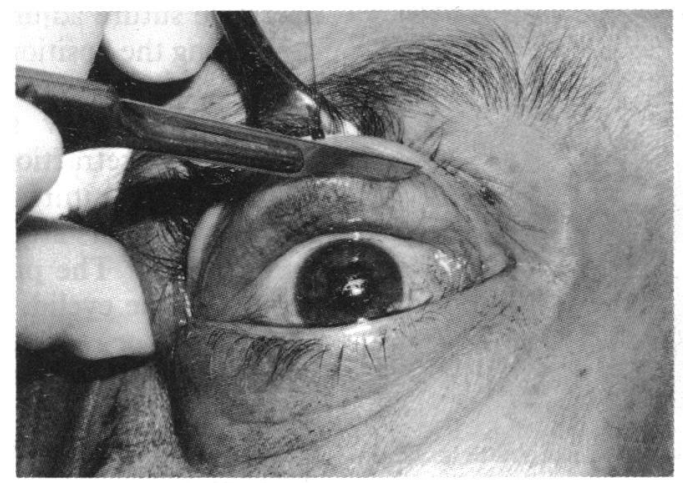

excessive upper lid retractor recession for thyroid associated lid retraction (three), and one case following two previous ptosis repairs. These were all operated via the anterior approach. One case of neurogenic ptosis in an adult was repaired via the posterior approach. There were seven patients with lid retraction. Five were lowered by the anterior approach; thyroid associated lid retraction (three), post-excessive ptosis correction (two), one of whom had an ocular myopathy. Two patients with thyroid-associated lid retraction had their eyelids lowered by the posterior approach. In eight of these 15 patients, no postoperative adjustment was required and the sutures were simply tied at 24 hours. In the other seven patients, one or more sutures were adjusted to raise or lower part or all of the eyelid. The only complication has been a mild inflammatory reaction in the skin around the sutures in two patients. If the knot is still present 4 weeks postoperatively, it is cut and removed with any attached suture. The longest follow up is only 8 months, but there has been no unexpected late change in eyelid contour or height in any of the 15 patients to date.

\section{Discussion}

In ptosis surgery under local anaesthetic, most surgeons will set the height of the lid by (a series of) intraoperative estimates and revisions. The patient may be asked to sit up and the height of one or both lids assessed. Care may be taken to minimise the amount of local anaesthetic injected in the region of the levator muscle and allowance is made for the temporary paresis of the orbicularis. Preoperative measurements of levator function, amount of ptosis, and aetiology (myopathic or neuropathic) are taken into consideration with the intraoperative lid position. In spite of these proved, time honoured techniques there is still a significant rate of inaccuracy in ptosis surgery via the anterior ${ }^{2}$ and posterior $^{3}$ approaches, and a means of postoperative adjustment is desirable. This applies in particular to neurogenic ptosis in which the results are more difficult to predict, and in patients with increased risk of corneal exposure - ocular myopathies, poor Bell's phenomenon, and corneal hypoaesthesia.

The unpredictability of thyroid lid surgery is well known among oculoplastic surgeons with under and overcorrections a recurring problem. The amount of lid lowering performed is determined by the preoperative assessment of lid retraction, and intraoperative lid height and assessment of residual lid restriction after retractor release. Release of the retractors without spacers has been shown to be as effective and with results as predictable as with the use of spacers; and insertion of foreign material including non-autogenous sclera is avoided. ${ }^{4}$ This nonrequirement of spacers facilitates the use of adjustable sutures since the levator tarsus distance is no longer fixed by a spacer and can be altered postoperatively. The sutures recessing the lid retractors are in fact 'hang back' sutures. Similar principles apply to correction of lid retraction from other causes - for example, facial nerve palsy. 

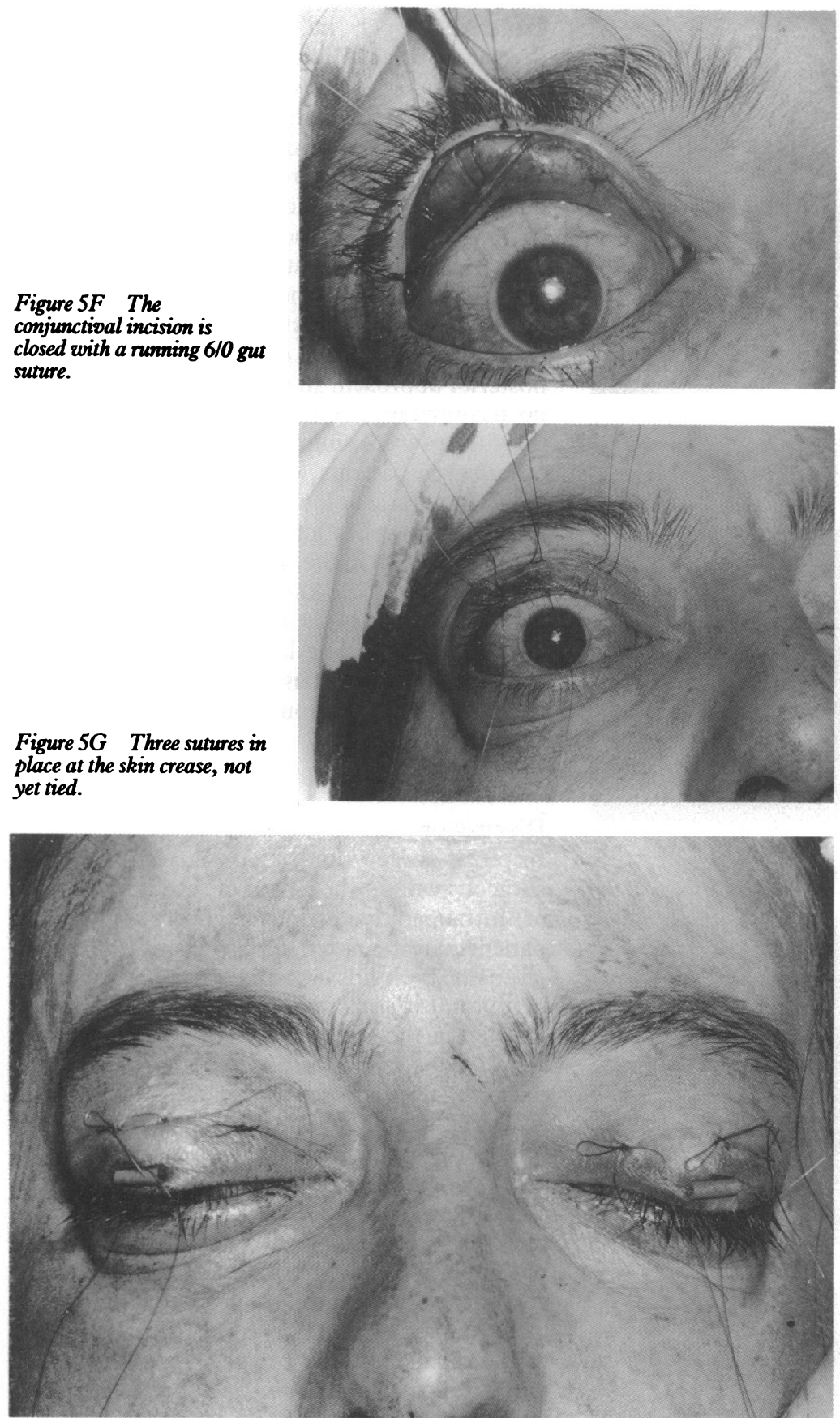

Figure $5 H$ Three adjustable sutures in position. Note reverse Frost sutures which will be taped to the patient's cheeks. operative suture adjustment of lid height while controlling the position of the skin crease has not been performed previously to the authors' knowledge in non-brow suspension correction of ptosis and lid retraction.

Adjustable sutures in strabismus surgery became popular after their development by Jampolsky. ${ }^{14}$ is The principles are similar when applied to the eyelid. The muscle is able to be advanced to or recessed from the muscle insertion point with the sutures passing through the tarsal plate instead of a scleral tunnel. In eyelid retraction, the retractors are suspended from their insertion with a hang back suture. Following surgery, the retractors will then heal in their new position. Our choice of suture is a $5 / 0$ coated absorbable polyglycolic acid Vicryl suture but nylon or Prolene can also be used. The suture can be knotted at the levator muscle to avoid the suture slipping when adjusting either end. This prevents suture removal, however, and requires an absorbable suture. Uncoated polyglycolic acid dexon has more tissue drag and is therefore slightly less easy to adjust compared with Vicryl. Vicryl is used since the knot holds well, has good tensile strength, and travels through tissue adequately when adjusting. Seesawing the suture through the tarsal plate to reduce friction within the suture tract was not found to be necessary nor was the injection of corticosteroid required to delay healing and facilitate adjustment. The bow knot has been used successfully in all procedures in preference to the sliding handle knot used in strabismus surgery. ${ }^{16}$ In adjustable strabismus surgery, Healon has been used to facilitate muscle adjustment, but this has not yet been necessary in eyelid surgery. ${ }^{17} \mathrm{~A}$ reasonable length of suture should be left at the end of surgery for adjustment. A traction suture through the upper lid margin for countertraction in adjustment was not found to be necessary in ptosis surgery.

The difficulty with the development of adjustable upper lid sutures in raising or lowering a lid has been twofold - the alteration of the position of the skin crease with suture adjustment, and the adjustability of the upper lid retractors postoperatively. The skin crease challenge is solved by placing the sutures through the anterior surface or edge of the tarsal plate in the anterior approach. In setting the height of the skin crease, the vertical height of the tarsal plate must be within 1-2 mm of the height of the skin crease. This may not be possible in patients with a high skin crease (or small tarsal plate). In setting the lid height, since the sutures pass through the tarsus within 1-2 mm of the desired skin crease, adjustment of the lid height will not alter the height of the skin crease. In the posterior approach, it is technically easiest to set the skin crease within 1-2 mm of the edge of the upper border of the tarsal plate.

In the correction of lid retraction, no tarsus is excised, since this would reduce the lid lowering effect of the procedure. The sutures are brought out through the skin, level with the upper border of the tarsal plate. Since the normal skin crease is usually 6-7 $\mathrm{mm}$ from the lid margin, the skin crease rises, but if the retractor recession is bilateral, symmetry is maintained and this does tion of ptosis but involve opening the wound and resuturing the aponeurosis. ${ }^{7}$ Methods for postoperative adjustment of brow suspension surgery have been reported ${ }^{8-13}$ but true post- 


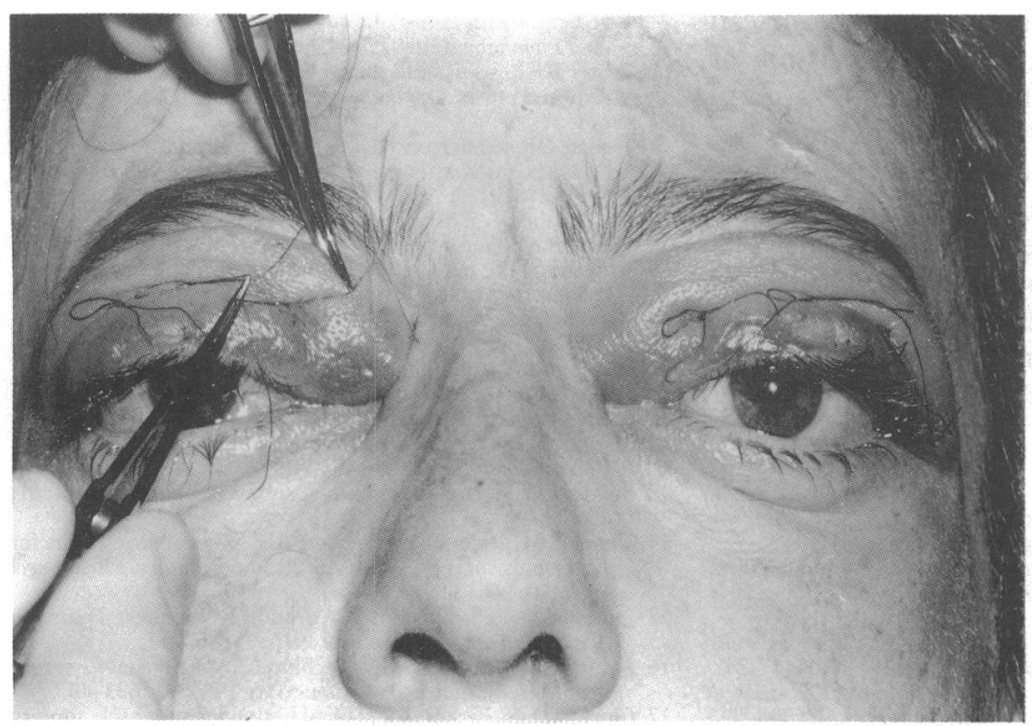

Figure 5I Lid position at 24 hours. The right upper lid is slightly low and is elevated postoperatively. discomfort. The lid height may be altered if the swollen lid alters the amount of retractor recession - that is, the levator tarsus part of the suture may be effectively shortened and the skin tarsus part lengthened with swelling in the anterior pretarsal part of the lid. This would reduce the amount of recession in lid retraction or amount of lid lift in ptosis. This has not been found to be a problem, probably because the pulling action of the levator complex keeps the suture in the desired position. Nevertheless, intraoperative haemostatis and firm postoperative padding are recommended to reduce postoperative lid swelling.

The anterior and posterior approaches used in ptosis and thyroid lid retraction both achieve satisfactory results. The anterior approach may be more desirable to many surgeons since it is the more common technique for ptosis surgery. It tends to form a harsher skin crease than the posterior approach. Posterior approach ptosis surgery may be faster for simple aponeurotic advancement when the desired skin crease is within $1 \mathrm{~mm}$ of the upper border of the tarsal plate. It has the advantage of being able to tie the sutures over a bolster rather than over the wound. It is not possible to perform, however, in extreme cases of thyroid lid retraction in which the tarsus cannot be everted.

Any case that can be adjusted postoperatively under local anaesthetic, may have adjustable sutures with slip knotting of the sutures performed. The sutures are easily permanently tied postoperatively without alteration of an already satisfactory lid position, since the suture is held where it passes through the tarsal plate. Since the technique of adjustable sutures offers postoperative adjustment, the results of ptosis and lid retraction surgery should be as good whether the surgery is performed under general or local anaesthesia.

A possible application of adjustable sutures is ptosis surgery performed under general anaesthesia in older children since ptosis surgery under general anaesthesia is less predictable. If revision is required, then the lid is easily and quickly adjusted without opening the wound. Even if a second general anaesthetic was required, subsequent adjustment is easier than complete revision of the surgery. In younger children, if adjustable sutures are used, they would need to be well tied since it is anticipated that adjustment is not required in most cases. The suture ends would also need to be left short so that the child's fingers can't pull on them but even then there is the significant risk of slippage or undoing of a knot that is bow knotted. The use of adjustable sutures in young children is therefore not recommended.

The value of being able to adjust the eyelid height and contour very simply postoperatively without affecting the skin crease level makes it worth presenting the techniques at this early stage. The method is a useful adjunct to ptosis and lid retraction surgery and is recommended for those cases in which it is notoriously difficult to predict the postoperative lid level, such as traumatic ptosis, thyroid associated lid retraction, and ptosis correction after excessive upper lid lowering, etc. The techniques are a simple
Postoperative swelling of the lid may make accurate lid height assessment and adjustment difficult and lid oedema will tend to ptose the lid. Adjustment, however, was easily performed in all cases to date and with minimum or no patient 
modification of the standard procedures, and can be easily adopted by any surgeon capable of performing ptosis and lid retraction surgery. They do not replace the standard techniques, but are intended to give an added measure of postoperative control of the lid level which is valuable in specific circumstances, as discussed. The maximum follow up to date is only 8 months, and although the results so far are extremely satisfactory, it will be necessary to reassess the results in the future after a longer follow up period.

1 Collin JRO. A manual of systematic eyelid surgery. 2nd ed. Edinburgh: Churchill Livingstone, 1989.

2 Tyers AG, Collin JRO. Senile ptosis - introduction and anterior approach. Trans Ophthalmol Soc UK 1984; 104: anterior.

3 Collin JRO, Tyers AG. Senile ptosis - posterior approach and complications. Trans Ophthalmol Soc UK 1984; 104: 17-21.

4 Older JJ. Surgical treatment of eyelid retraction associated with thyroid eye disease. Ophthalmic Surg 1991; 22: 318-22.

5 Hyklema HA, Koornneef $L$. Treatment of ptosis by levator resection with adjustable sutures via the anterior approach. Br f Ophthalmol 1989; 73: 416-8.

6 Berris CE. Adjustable sutures for the correction of adult acquired ptosis. Ophthalmic Plastic Reconstr Surgery 1988; 4 $171-3$.

7 Jordan DR, Anderson RL. A simple procedure for adjusting eyelid position after ptosis surgery. Arch Ophthalmol 1987 105: 1288-91.

8 Argamaso RV. The adjustable fascia lata sling for the correction of blepharoptosis. Br F Plast Surg 1974; 27: 274-5.

9 Argamaso RV, Lewin ML. Fascia lata sling in blepharoptosis: enhancement of result by postoperative adjustment. 7 Paediat Ophthalmol 1976; 13: 51-5.

$10 \mathrm{Akyol} \mathrm{N}$, able suture technique. Acta Ophthalmol 1990; 68: 487-90.

11 Jampolsky A. Adjustable eyelid-brow suspension. Am $\mathcal{F}$ Ophthalmol 1979; 88: 109-12.

12 Mustarde JD. Problems and possibilities in ptosis surgery Plast Reconstr Surg 1975; 56: 381-8.

13 Snyder CC, Norton W. Eyelid ptosis. Plast Reconstr Surg 1961 27: 586-96.

14 Thorson JC, Jampolsky A, Scott AB. Topical anaesthesia for strabismus surgery. Trans Am Acad Ophthalmol Otolaryngol 1966; 70: 968-72.

15 Jampolsky A. Strabismus reoperation techniques. Trans Am Acad Ophthalmol Otolaryngol 1975; 79: 704-17.

16 Kraft SP, Jacobson ME. Techniques of adjustable suture strabismus surgery. Ophthalmic Surg 1990; 21: 633-40.

17 Clorfeine GS, Parker WT. Use of healon in eye muscle surgery Clorfeine GS, Parker WT. Use of healon in eye muscle surgery
with adjustable sutures. Ann Ophthalmol 1987; 19: 215-7. 